\title{
Special Issue on Mobile Sensor Networks: Advanced Technologies and Their Applications
}

\author{
Hyuncheol Kim ${ }^{1}$ Donghwi Lee ${ }^{2} \cdot$ Wonhyung Park ${ }^{3}$
}

Published online: 17 March 2017

(C) Springer Science+Business Media New York 2017

Mobile cloud computing has been studied in various fields as a paradigm that utilizes computing resources such as computing power, memory, and storage device of cloud computing to solve the resource constraint problem of the mobile device. Because mobile devices operate on batteries and are equipped with energy-efficient processors, it is difficult to perform complex calculations. Also, in the case of wireless communication, the bandwidth is limited depending on the characteristics of the connected communication module. Thus, in applications such as image processing that require significant computing power and communication bandwidth, it is more efficient to utilize offloading techniques that delegate tasks to the cloud and transmit only results, rather than performing operations on mobile devices.

Mobile cloud sensor networks have been proposed for research and use in new challenging applications with advanced technologies, such as healthcare, plant factory, bio-IT, medical-IT, military surveillance, and scientific exploration. To serve without any problems there are many challenging issues: mobility, IoT, wireless sensor network, monitoring/management mechanism, etc. However, various studies have been conducted on mobile cloud sensor networks, but there are still many problems to be solved.

This special issue included nine carefully selected papers and covers high-quality and state-of-the-art research issues and results regarding the mobile cloud sensor network technologies. In particular, the special issue is going to showcase the most recent achievements and developments in the realm of mobile cloud sensor networks.

Hyuncheol Kim

hckim@nsu.ac.kr

Donghwi Lee

dhclub@dsu.ac.kr

Wonhyung Park

whpark02@gmail.com

1 Namseoul University, Cheonan, South Korea

2 Dongshin University, Naju, South Korea

3 Far East University, Umsung, South Korea 
Three papers discuss security issues in mobile cloud sensor networks. Choi et al.'s paper proposes a web application acquisition procedure for outsourcing that can provide safe web services by strengthening security and makes possible to efficiently manage software by outsourcing from the plan for web applications. Park et al.'s paper present a more improved approach, in the perspective of cost and requirement satisfaction. The proposed method mainly exploits luminance areas and pixel location, so that the produced image identifiers could satisfy the one-to-one relationship between images and identifiers. Teh et al.'s paper develop a prototype that utilizes an identity-based identification scheme to provide access control for incoming and outgoing personnel from the building, as well as their activities within.

Three papers discuss applied services and management of mobile cloud sensor networks. Cho et al.'s paper proposes an end-to-end network performance management framework based on case-based reasoning with the case library and multi-agent integrated with perfSONAR. Anjum et al.'s paper provides taxonomy on communication strategies of MANETs during disaster and emergency situation. Chantaraskul et al.'s paper proposes a mechanism called safety communication based adaptive multi-channel assignment, which allows flexible multi-channel usage based on real-time communication traffic condition.

Finally, three articles deal with hardware, frequency hopping, and energy management of mobile cloud sensor networks. Kim et al.'s paper propose a user-oriented load balancing scheme for an energy-efficient load balancing in wireless networks which are based on allocating load on wireless sensor nodes proportionally to each of the agent's capacity and user-oriented approach. Hu et al.'s paper proposes a scheme to take advantage of compressive sensing for sparse signal in frequency domain. Baek et al.'s paper represents a dynamically-linked library (DLL) wrapping approach to support the KHR debug extension even on the old OpenGL ES 1.1 versions.

At last, guest editors would like to take this opportunity to thank all the authors of the special issue for their excellent contributions, and reviewers, for their treasurable comments. Springer publishing staff is also acknowledged for their kindness, swiftness, and enthusiasm for this issue.

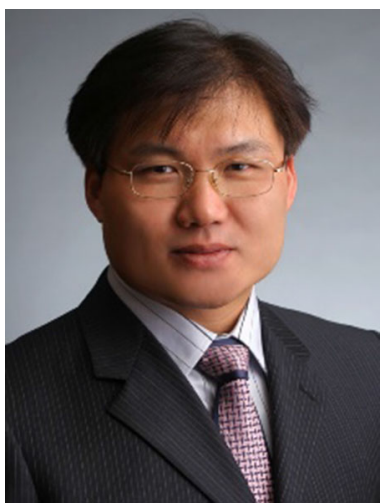

Hyuncheol Kim received his $\mathrm{Ph}$. D. degree in the department of information engineering from Sungkyunkwan University, Korea, in 2005. From 1992 to 2002, he was a senior engineer in Electronics and Telecommunications Research Institute (ETRI), Daejeon, Korea. He has been a professor in the department of computer science at Namseoul University, Korea. His research interests include cloud computing, next generation network and embedded computing systems. 


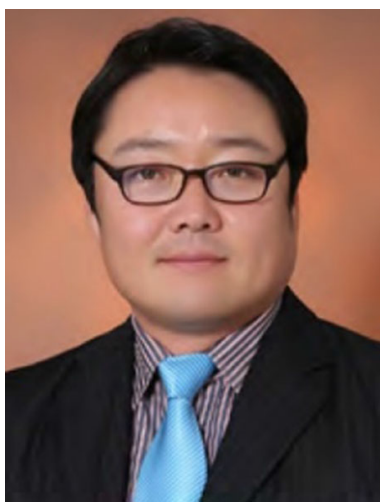

Donghwi Lee received the Ph.D. degree in Information Security from Kyonggi University, Korea, in 2001 and 2007. Research Scholar of the University of Colorado Denver in 2010 and 2011, USA, He is currently a Professor of DongShin University, His research areas include Information Security and Control System Security.

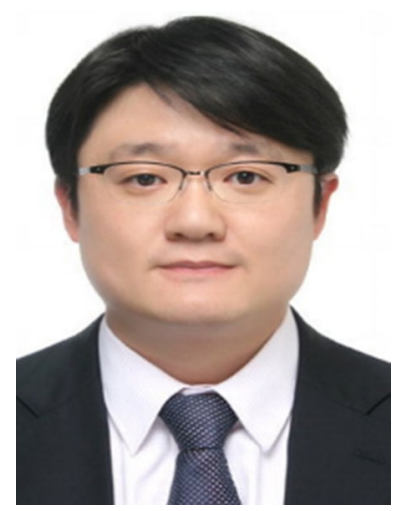

Wonhyung Park received the B.S. and M.S. degree in Industrial Engineering from Seoul National University of Science and Technology, Korea in 2002 and 2005. He received Ph.D. degrees in Department of Information Security from the Kyonggi University, South Korea, in 2009. Now, he is Professor in Department of Industrial Security, Far East University, South Korea. 